Andreas Krebs, Jürgen Doerfer, Sarah Catherina Grünert, Jan Wöhrl, Bernhard Stier, Arno Schmidt-Trucksäss, Kai Lichte, Karl Winkler, Jürgen Grulich-Henn, Martin Holder and Karl Otfried Schwab*

\title{
Decreased levels of homoarginine and asymmetric dimethylarginine in children with type 1 diabetes: associations with cardiovascular risk factors but no effect by atorvastin
}

\section{Abstract}

Objectives: To investigate homoarginine and asymmetric dimethylarginine (ADMA) in controls compared to children with type 1 diabetes (T1D) and if homoarginine and ADMA are affected by atorvastatin.

Methods: Homoarginine and ADMA levels of 28 T1D patients were compared to levels of 41 controls. In T1D patients, homoarginine and ADMA were determined at baseline, 1 year, and 2 years at daily $10 \mathrm{mg}$ atorvastatin or placebo within a double-blind study.

Results: At baseline, both homoarginine and ADMA were lower $(\mathrm{p}<0.001)$ in T1D patients compared to controls. In T1D patients, homoarginine and ADMA were not influenced by atorvastatin. Inverse correlations between homoarginine and HbA1c $(\mathrm{p}<0.001)$ and between ADMA and systolic blood pressure $(\mathrm{p}=0.005)$ and pulse pressure ( $\mathrm{p}=0.003)$ were shown.

*Corresponding author: Karl Otfried Schwab, MD; Department of Pediatrics and Adolescent Medicine, University Medical Center, Freiburg, Germany, Phone: +49-761-270-44821,

Fax: +49-761-270-44140,

E-mail: karl.otfried.schwab@uniklinik-freiburg.de

Andreas Krebs, Jürgen Doerfer and Sarah Catherina Grünert:

Department of Pediatrics and Adolescent Medicine, University Medical Center, Freiburg, Germany

Jan Wöhrl and Karl Winkler: Department of Clinical Chemistry, University Medical Center, Freiburg, Germany

Bernhard Stier: Practice for Pediatrics and Adolescent Medicine, Butzbach, Germany

Arno Schmidt-Trucksäss: Institute of Exercise and Health

Sciences, Department Sports Medicine, University of Basel, Basel, Switzerland

Kai Lichte: Clinic for Pediatrics and Adolescent Medicine, Schwarzwald-Baar Medical Center, Villingen-Schwenningen, Germany

Jürgen Grulich-Henn: Department of Pediatrics and Adolescent Medicine, University Medical Center, Heidelberg, Germany

Martin Holder: Children's Hospital, Olgahospital, Stuttgart, Germany
Conclusions: Homoarginine and ADMA levels are decreased and associated with cardiovascular risk factors in children with T1D without being affected by atorvastatin.

Keywords: ADMA; atorvastatin; homoarginine; pediatric type 1 diabetes.

DOI 10.1515/jpem-2014-0083

Received February 19, 2014; accepted July 14, 2014; previously published online August 15, 2014

\section{Introduction}

L-homoarginine (HoArg) is an amino acid supposed to be formed by enzymatically transamination of L-lysine using L-arginine:glycine-amidinotransferase $(1,2)$. Because HoArg shows structural similarity to L-arginine, it can serve as a substrate or act as an inhibitor for nitric oxide (NO) synthesis thus increasing or decreasing NO production in the endothelium $(3,4)$. In adults with cardiovascular disease or type 2 diabetes, low HoArg has been shown to be associated with cardiovascular morbidity $(5,6)$ In children and adolescents with type 1 diabetes (T1D) who are known to be at risk for developing premature atherosclerosis $(7,8)$, data on HoArg plasma concentrations are not available in the literature. Asymmetric dimethylarginine (ADMA) is an endogenous form of the amino acid arginine and exhibits potent inhibitory activities on endothelial nitric oxide synthases (eNOS) (9). Studies have shown that plasma ADMA can serve as a risk marker for endothelial dysfunction and cardiovascular disease $(10,11)$, but inconsistent data exist regarding the role of ADMA in patients with T1D $(12,13)$. In terms of medical treatment, statin therapy has shown contradictory effects on ADMA levels in adult patients with vascular disease 
(14, 15), but therapy data as to how HoArg and ADMA are affected by statins in children with T1D are currently not available. The present article is based on a 2-year, double-blind, placebo-controlled study with atorvastatin investigating associations of HoArg and ADMA with diabetes-related and cardiovascular risk factors in children with T1D.

\section{Materials and methods}

This investigation was conducted as part of the clinical trial entitled Vasoprotection by Atorvastatin in Children and Adolescents with Type 1 Diabetes, a 2-year pilot study (ATV-D-03-005G). The protocol was approved by the Ethics Committee at the Albert-Ludwigs-University of Freiburg (registration number: 243/03; German Clinical Trials Register: DRKS00000297, Registry URL: http://www.germanctr.de), and all participants or their parents signed an informed consent.

\section{Study and participants}

The study was a randomized, double-blind, placebo-controlled clinical trial. From 2005 to 2010, protocol-specified enrolment, monitoring, and treatment administration of study participants were carried out. Children with T1D $(n=28)$ were randomly allocated to receive either $10 \mathrm{mg}$ atorvastatin $(\mathrm{n}=18)$ or placebo $(\mathrm{n}=10)$. There were six dropouts due to one fatal traffic accident and five cases of noncompliance because of poor glycemic control with HbA1c $>9.5 \%$ measured twice within a period of 4 weeks, inadequate intake of the study medication, or failure to attend the study visits. For ethical reasons and to avoid unnecessary use of atorvastatin therapy, only diabetic children with carotid intima-media thickness values (A. Schmidt-Trucksäss) above the 75th percentile were included (7). T1D was diagnosed according to the American Diabetes Association (16). For comparison, 41 healthy age- and sex-matched controls were collected during a systematic health preventative check-up at a practice for pediatrics and adolescent medicine. None of the controls received any medical therapy. In children with T1D, only specific diabetic treatment and study medication were allowed. Further characteristics of controls and patients are summarized in Table 1.

\section{Clinical and laboratory examinations}

Standard deviation scores for body mass index $\left(\mathrm{kg} / \mathrm{m}^{2}\right)$ were calculated using corresponding normative data for German children (17). Blood pressure was measured as the average of three measurements using the Dinamap pediatric monitor (Critikon, Tampa, FL, USA) with the appropriate cuff size for the upper arm. Brachial pulse pressure was calculated as the difference between systolic and diastolic blood pressure. Albumin in urine was determined in children with T1D on first morning urine samples (BN ProSpec system, Siemens Healthcare Diagnostics, Marburg, Germany) taken at the end of the follow-up period. Serum creatinine was measured (Roche Cobas System 8000, Module c702, Roche Diagnostics $\mathrm{GmbH}$, Mannheim, Germany) in children with T1D at baseline and at the end of the follow-up period. Evaluation and monitoring of glycemic status was achieved by the determination of HbA1c which was standardized according to the Diabetes Control and Complications Trial (DCCT) reference range of 4.05-6.05\% (18).

Blood samples were taken after an overnight fast for arginine, HoArg, and ADMA and analyzed using stable isotope dilution techniques and liquid chromatography-tandem mass spectrometry (LC20A Shimadzu prominence, Duisburg, Germany; AB Sciex 4000 QTRAP, Darmstadt, Germany). Blood was placed in prechilled heparinized tubes and immediately spun down at $4^{\circ} \mathrm{C}$. The plasma was deproteinized using perchloric acid (2:1) and spun down, after which the supernatant was transferred and stored at $-80^{\circ} \mathrm{C}$ until analysis. Separation was done by liquid chromatography on a $150-\mathrm{mm} \times 3-\mathrm{mm}$

Table 1 Baseline characteristics of children and adolescents with type 1 diabetes compared to healthy controls supplemented by plasma levels of arginine, ADMA, and homoarginine.

\begin{tabular}{|c|c|c|c|}
\hline \multirow[t]{2}{*}{ Parameters } & \multicolumn{2}{|r|}{ Participants } & \multirow[t]{2}{*}{$\mathrm{p}$-Value } \\
\hline & $\begin{array}{r}\text { Type } 1 \text { diabetes } \\
(\mathrm{n}=\mathbf{2 8}, \mathbf{2 7 \%} \text { female) }\end{array}$ & $\begin{array}{r}\text { Controls } \\
(n=41,34 \% \text { female })\end{array}$ & \\
\hline Age, years & $14.5(13.7-15.0)$ & $14.0(13.5-14.0)$ & NS \\
\hline $\mathrm{HbA} 1 \mathrm{c}, \%$ & $8.3(7.8-8.7)$ & - & - \\
\hline Diabetes duration, years & $5.5(4.7-7.6)$ & - & - \\
\hline Insulin, IU/kg/day & $0.83(0.73-0.96)$ & - & - \\
\hline $\mathrm{BMI}, \mathrm{kg} / \mathrm{m}^{2}$ & $22.9(20.3-25.3)$ & $20.0(19.1-21.1)$ & NS \\
\hline BMI-SDS & $0.79(-0.04-1.51)$ & $0.32(-0.16-0.5)$ & NS \\
\hline $\mathrm{SBP}, \mathrm{mmHg}$ & $121(115-125)$ & $115(112-118)$ & 0.015 \\
\hline $\mathrm{DBP}, \mathrm{mmHg}$ & $70(67-73)$ & $70(66-71)$ & NS \\
\hline Pulse pressure, $\mathrm{mmHg}$ & $51(46-55)$ & $45(43-48)$ & NS \\
\hline Arginine, $\mu \mathrm{mol} / \mathrm{L}$ & $129.4(121.1-149.0)$ & $138.2(128.9-150.2)$ & NS \\
\hline ADMA, nmol/L & $399.7(357.4-478.9)$ & $1059.5(996.0-1179.8)$ & $<0.001$ \\
\hline Homoarginine, $\mu \mathrm{mol} / \mathrm{L}$ & $0.900(0.899-1.053)$ & $1.420(1.360-1.886)$ & $<0.001$ \\
\hline
\end{tabular}

Data are medians (95\% CI); BMI-SDS, body mass index-standard deviation score; SBP, systolic blood pressure; DBP, diastolic blood pressure; ADMA, asymmetric dimethylarginine; a Mann-Whitney U test; NS, not significant. 
silica column with an isocratic mobile phase consisting of water, acetonitrile, trifluoroacetic acid, and propionic acid (10:90:0.025:1 by volume) with a chromatographic run time of $7 \mathrm{~min}$, as reported previously $(2,19)$.

\section{Statistical analyses}

Data are presented as median with $95 \%$ confidence intervals (CIs). Differences between groups were analyzed by the non-parametric Mann-Whitney rank sum test (U test). Changes from baseline to the 1-year and 2-year follow-up assessments were analyzed by means of the Friedman test. Correlation analyses were performed using the Spearman rank correlation coefficients ( $r$, two-tailed) for exploring associations between two variables. Analysis of covariance (ANCOVA) was used to determine associations between atorvastatin, HoArg, and ADMA. Significant differences were assumed for $\mathrm{p}<0.05$. The statistical analyses were carried out with commercially available software (SPSS version 20.0; Chicago, IL, USA). In addition, post hoc power analyses were performed using the non-commercial $\mathrm{G}^{\star}$ Power 3.1.7 software for Windows (Heinrich Heine University, Düsseldorf, Germany; www.psycho.uni-duesseldorf.de/abteilungen/ aap/gpower3).

\section{Results}

Basic characteristics of children with and without T1D are shown in Table 1. Regarding the sex distribution, there was no statistically significant difference between children with and without T1D ( $\mathrm{p}=0.115)$. In children with T1D, urinary albumin level at the end of the follow-up period was $0.35 \mu \mathrm{mol} / \mathrm{L}(2.4 \mathrm{mg} / \mathrm{dL})$ in patients receiving atorvastatin compared to $0.81 \mu \mathrm{mol} / \mathrm{L}(5.6 \mathrm{mg} / \mathrm{dL})$ in placebo-treated patients $(\mathrm{p}=0.50)$, based on the defined normal value $<2.17 \mu \mathrm{mol} / \mathrm{L}$ ( $15 \mathrm{mg} / \mathrm{dL}$ ). Serum creatinine was measured in children with T1D at baseline [atorvastatin group $=63.65 \mu \mathrm{mol} / \mathrm{L}(0.72 \mathrm{mg} / \mathrm{dL})$ versus placebo group $=58.35 \mu \mathrm{mol} / \mathrm{L}(0.66 \mathrm{mg} / \mathrm{dL}), \mathrm{p}=0.69]$ and at the end of the follow-up period [atorvastatin group $=70.72 \mu \mathrm{mol} / \mathrm{L}$ $(0.80 \mathrm{mg} / \mathrm{dL})$ versus placebo group $=69.84 \mu \mathrm{mol} / \mathrm{L}(0.79$ $\mathrm{mg} / \mathrm{dL}), \mathrm{p}=1.00]$. None of the enrolled children with T1D had a kidney disorder.

Post hoc analyses were performed for power calculation to detect differences of ADMA or HoArg between the two independent baseline groups ( 28 children with T1D versus 41 children without diabetes) at a significance level of $\alpha=0.05$. The power (1- $\beta$ error probability) was $100 \%$ for ADMA and $94 \%$ for HoArg.

At baseline and prior to atorvastatin therapy, HoArg and ADMA were significantly lower in children with T1D compared to controls (Table 1). In T1D subjects, inverse correlations were found between HoArg and HbA1c $(r=-0.677, p<0.001)$ and between ADMA and HbA1c $(\mathrm{r}=-0.351, \mathrm{p}=0.085)$; systolic blood pressure $(\mathrm{r}=-0.548$, $\mathrm{p}=0.005)$; and pulse pressure $(\mathrm{r}=-0.577, \mathrm{p}=0.003)$. No significant correlations were found between further cardiovascular risk factors (age, BMI-SDS, diabetes duration, LDL cholesterol, HDL cholesterol, triglycerides) and ADMA or HoArg.

The 2-year atorvastatin therapy did not have any significant influence on plasma concentrations of HoArg and ADMA (Table 2). Analysis of covariance (ANCOVA) revealed no association between atorvastatin and HoArg $(F=3.060, \mathrm{p}=0.096)$ or ADMA $(F=0.073, \mathrm{p}=0.789)$.

\section{Discussion}

One of the earliest stages in the development of atherosclerosis is endothelial cell dysfunction which is characterized by reduced bioavailability of NO formed from arginine through eNOS in the vascular endothelium (20). Relevant key factors for diabetes-related endothelial dysfunction are insulin deficiency and hyperglycemia (12). Most recently, there have been various publications indicating that decreased plasma concentrations of HoArg may play an important role as a cardiovascular risk marker $(3,5,6)$. But the question whether and how HoArg is involved in diabetes-related endothelial dysfunction still needs to be answered. So far, it is known that HoArg can be involved in insulin release which in turn acts as an activator of eNOS to convert arginine into NO and citrulline (21). In our investigation, children and adolescents with T1D had significantly lower HoArg levels than healthy controls possibly suggesting their increased cardiovascular risk. Interestingly, in another study with 40 healthy children and adolescents, the same median plasma homoarginine concentration was measured as in our controls. An association between intimamedia thickness or extramedial thickness of common carotid arteries and homoarginine could not be found (22). In general, children with T1D however are at high risk of premature, subclinical atherosclerosis which has been confirmed by providing evidence of endothelial dysfunction and increased intimamedia thickness ( 7 , 23). One particularly interesting aspect of our study is the inverse correlation between HoArg and HbA1c in children with T1D which leads to the thought that there are close links between HoArg and glucose metabolism. Chronic hyperglycemia is one of numerous risk factors affecting endothelial function (24) and is very evidently accompanied by low HoArg plasma levels. Because it is a fact, that HoArg is an alternative substrate for eNOS, an animal study is instructive revealing improved NO bioavailability 
Table 2 L-arginine, asymmetric dimethylarginine, and L-homoarginine in children with type 1 diabetes at baseline, 1 year, and 2 years after therapy with atorvastatin or placebo.

\begin{tabular}{|c|c|c|c|c|c|c|c|c|}
\hline \multirow[t]{2}{*}{ Parameter } & \multicolumn{4}{|c|}{ Atorvastatin $(10 \mathrm{mg} /$ day $)$} & \multicolumn{4}{|r|}{ Placebo } \\
\hline & $\begin{array}{r}\text { Baseline } \\
(n=18)\end{array}$ & $\begin{array}{l}1 \text { year } \\
(n=16)\end{array}$ & $\begin{array}{l}2 \text { years } \\
(n=14)\end{array}$ & $p$-Value & $\begin{array}{r}\text { Baseline } \\
(n=10)\end{array}$ & $\begin{array}{r}1 \text { year } \\
(n=9)\end{array}$ & $\begin{array}{r}2 \text { years } \\
(\mathrm{n}=8)\end{array}$ & p-Value \\
\hline Arginine, $\mu \mathrm{mol} / \mathrm{L}$ & $\begin{array}{r}128.2 \\
(110.8-155.9)\end{array}$ & $\begin{array}{r}128.2 \\
(111.8-161.6)\end{array}$ & $\begin{array}{r}113.8 \\
(97.7-137.1)\end{array}$ & NS & $\begin{array}{r}133.1 \\
(119.5-158.2)\end{array}$ & $\begin{array}{r}153.1 \\
(111.8-190.6)\end{array}$ & $\begin{array}{r}158.0 \\
(102.7-184.3)\end{array}$ & NS \\
\hline ADMA, nmol/L & $\begin{array}{r}422.1 \\
(395.4-495.1)\end{array}$ & $\begin{array}{r}422.5 \\
(391.2-481.6)\end{array}$ & $\begin{array}{r}407.0 \\
(373.6-452.3)\end{array}$ & NS & $\begin{array}{r}399.7 \\
(356.2-516.1)\end{array}$ & $\begin{array}{r}410.7 \\
(356.2-524.0)\end{array}$ & $\begin{array}{r}397.4 \\
(326.9-483.2)\end{array}$ & 0.034 \\
\hline Homoarginine, $\mu \mathrm{mol} / \mathrm{L}$ & $\begin{array}{r}1.000 \\
(0.893-1.121)\end{array}$ & $\begin{array}{r}0.900 \\
(0.852-1.085)\end{array}$ & $\begin{array}{r}0.900 \\
(0.797-1.017)\end{array}$ & NS & $\begin{array}{r}0.900 \\
(0.852-1.104)\end{array}$ & $\begin{array}{r}1.0 \\
(0.867-1.133)\end{array}$ & $\begin{array}{r}1.050 \\
(0.860-1.240)\end{array}$ & NS \\
\hline LDL cholesterol, $\mathrm{mg} / \mathrm{dL}^{\mathrm{b}}$ & $\begin{array}{r}85.0 \\
(77.2-104.7)\end{array}$ & $\begin{array}{r}63.5 \\
(48.4-75.6)\end{array}$ & $\begin{array}{r}54.5 \\
(48.1-70.8)\end{array}$ & $<0.01$ & $\begin{array}{r}85.0 \\
(70.2-98.7)\end{array}$ & $\begin{array}{r}75.0 \\
(58.2-96.9)\end{array}$ & $\begin{array}{r}84.0 \\
(72.4-106.9)\end{array}$ & NS \\
\hline HbA1c, \% & $\begin{array}{r}7.9 \\
(7.4-8.5)\end{array}$ & $\begin{array}{r}8.2 \\
(7.7-87)\end{array}$ & $\begin{array}{r}8.3 \\
(7.7-9.2)\end{array}$ & NS & $\begin{array}{r}8.2 \\
(7.0-9.0)\end{array}$ & $\begin{array}{r}7.8 \\
(6.9-8.9)\end{array}$ & $\begin{array}{r}8.7 \\
(7.5-9.3)\end{array}$ & NS \\
\hline
\end{tabular}

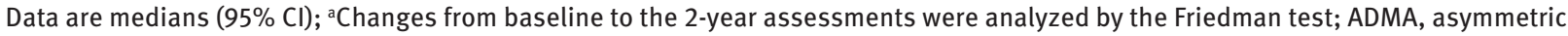
dimethylarginine; LDL, low-density lipoprotein; NS, not significant. ${ }^{\mathrm{b}}$ To convert values to $\mathrm{mmol} / \mathrm{L}$, multiply by a factor of 0.0259 .

by supplying arginine in hyperglycemic diabetic mice (25). Further investigations are warranted with particular regard to the role of HoArg in insulin and glucose regulation, and the potential of HoArg to act as a cardiovascular risk factor or serve as a clinically relevant diagnostic marker.

The mode of action of the eNOS-inhibitor ADMA as a risk marker for endothelial dysfunction and cardiovascular disease has been described in a large number of reports. In contrast to these investigations in non-diabetic adults where it was concluded that elevated ADMA levels are associated with subclinical atherosclerosis, cardiovascular events, and even total mortality (9-11), the importance of elevated ADMA in adult diabetic patients is far less clear due to diverse relationships with the metabolism of insulin and glucose, and diabetic vascular complications (12) Our particular aim was to determine clinical data, including long-term data regarding ADMA in children with T1D and to review the current literature on these subjects. In a study conducted in children and adolescents with T1D, it was demonstrated that ADMA plasma concentration was lower in diabetic than in healthy children, thereby probably activating atherogenic mechanisms by increased oxidative stress (13). We can also confirm from our own experience that ADMA concentration is significantly lower in children with T1D compared to healthy controls. Interestingly, it has been demonstrated that in human cultured endothelial cells ADMA accumulation was dose-dependently inhibited by insulin via changes in the activity of dimethylarginine-dimethylaminohydrolase (26). Furthermore, it was shown that both in young men with borderline hypertension and in adolescents and young adults with T1D ADMA concentration decreased significantly as the result of short-term insulin infusion $(27,28)$. Insulin-mediated increased cellular absorption or decreased release of ADMA and the improvement of hyperglycemia-induced down-regulation of the ADMAdegrading enzyme dimethylarginine-dimethylaminohydrolase by insulin have been discussed as main causes. In children with T1D, insulin absorption occurs independently of the current blood glucose level from the insulin depot in the subcutaneous tissue causing therapeutic, exogenous hyperinsulinemia which may thus contribute to their low ADMA levels.

In our children with T1D, the lowered endothelial parameters HoArg and ADMA did not change significantly in the course of the 2-year study. In placebo-treated patients, HoArg increased slightly and ADMA remained more or less at the same level. With regard to atorvastatin, there are some initial indications in the literature showing potential reduction of arterial stiffness in children with T1D (29). In the course of the effective atorvastatin treatment of our T1D patients, indicated by the significant reduction of LDL cholesterol, HoArg and ADMA showed only a small decrease. We think therefore that there is no simple causal relationship between these parameters, LDL cholesterol, and statin treatment. It is well known that statins also exert pleiotropic, lipidindependent effects such as increasing the expression of eNOS or reducing oxidative stress $(15,30)$ that could lead to an improvement of endothelial function. While no published data on HoArg could be found, ADMA levels, just as with our study, showed no significant reduction during statin treatment in various clinical trials (14, 30). However, a recent study in ischemic stroke patients 
revealed a significant relation between statin treatment and decreased ADMA levels (15).

In conclusion, decreased plasma levels of HoArg and ADMA in T1D children and their correlation with generally accepted cardiovascular risk factors may suggest that the two parameters are possibly associated with early atherosclerotic vascular changes. In the case of HoArg, there is an association with poor glycemic control that leads to hyperglycemia, whereas ADMA is correlated with systolic blood pressure, pulse pressure, and probably HbA1c. The 2-year atorvastatin therapy did not have any significant influence on comparatively low plasma concentrations of HoArg and ADMA in children with T1D. However, future long-term studies with larger patient numbers are warranted to confirm the role of HoArg and ADMA in T1D and atherosclerosis.

\section{Conflict of interest statement}

Funding: The study was supported by grants of Pfizer (Germany).

Conflict of interest: The authors declare that there is no duality of interest associated with this manuscript. The manuscript was exclusively developed by the participating authors without any fees.

\section{References}

1. Ryan WL, Wells IC. Homocitrulline and homoarginine synthesis from lysine. Science 1964;144:1122-7.

2. Atzler D, Mieth M, Maas R, Böger RH, Schwedhelm E. Stable isotope dilution assay for liquid chromatography-tandem mass spectrometric determination of L-homoarginine in human plasma. J Chromatogr B Analyt Technol Biomed Life Sci 2011;879:2294-8.

3. Davids M, Ndika JD, Salomons GS, Blom HJ, Teerlink T. Promiscuous activity of arginine:glycine amidinotransferase is responsible for the synthesis of the novel cardiovascular risk factor homoarginine. FEBS Lett 2012;586:3653-7.

4. Chatterjee A, Catravas JD. Endothelial nitric oxide (NO) and its pathophysiologic regulation. Vascul Pharmacol 2008;49:134-40.

5. März, W, Meinitzer A, Drechsler C, Pilz S, Krane V, et al. Homoarginine, cardiovascular risk, and mortality. Circulation 2010;122:967-75.

6. Drechsler C, Meinitzer A, Pilz A, Krane V, Tomaschitz A, et al. Homoarginine, heart failure, and sudden cardiac death in hemodialysis patients. Eur J Heart Fail 2011;13:852-9.

7. Krebs A, Schmidt-Trucksäss A, Doerfer, Grulich-Henn J, Holder M, et al. Cardiovascular risk in pediatric type 1 diabetes: sex-specific intima-media thickening verified by automatic contour identification and analyzing systems. Pediatr Diabetes 2012;13:251-8.

8. Schwab KO, Doerfer J, Hecker W, Grulich-Henn J, Wiemann D, et al. On behalf of the DPV initiative of the German Working Group for Pediatric Diabetology. Spectrum and prevalence of atherogenic risk factors in 27,358 children, adolescents, and young adults with type 1 diabetes. Cross-sectional data from the German diabetes documentation and quality management system (DPV). Diabetes Care 2006;29:218-25.

9. Vallance P, Leiper J. Cardiovascular biology of the asymmetric dimethylarginine: dimethylarginine dimethylaminohydrolase pathway. Arterioscler Thromb Vasc Biol 2004;24:1023-30.

10. Böger RH. Asymmetric dimethylarginine (ADMA) and cardiovascular disease: insights from prospective clinical trials. Vasc Med 2005;10:S19-25.

11. Maas R, Xanthakis V, Polak JF, Schwedhelm E, Sullivan LM, et al. Association of the endogenous nitirc oxide synthase inhibitor ADMA with carotid artery intima media thickness in the Framing. ham Heart Study Offspring Cohort. Stroke 2009;40:2715-9.

12. Anderssohn M, Schwedhelm E, Lüneburg N, Vasan RS, Böger RH. Asymmetric dimethylarginine as a mediator of vascular dysfunction and a marker of cardiovascular disease and mortality: an intriguing interaction with diabetes mellitus. Diab Vasc Dis Res 2010;7:105-18.

13. Huemer M, Simma B, Mayr D, Mühl A, Rami B, et al. Low levels of asymmetric dimethylarginine in children with diabetes mellitus type I compared with healthy children. J Pediatr 2011;158:602-6.

14. Valkonen V-P, Laakso J, Päivä H, Lehtimäki T, Lakka TA, et al. Asymmetrical dimethylarginine (ADMA) and risk of acute coronary events. Does statin treatment influence plasma ADMA levels? Arterioscler Suppl 2003;4:19-22.

15. Nishiyama Y, Ueda M, Otsuka T, Katsura K, Abe A, et al. Statin treatment decreased serum asymmetric dimethylarginine (ADMA) levels in ischemic stroke patients. J Atheroscler Thromb 2011;18:131-7.

16. Silverstein J, Klingensmith G, Copeland K, Plotnick L, Kaufman F, et al. Care of children and adolescents with type 1 diabetes. Diabetes Care 2005;28:186-212.

17. Kromeyer-Hauschild K, Wabitsch M, Kunze D, et al. Percentiles of body mass index in children and adolescents evaluated from different regional German studies. Monatsschr Kinderheilkd 2001;149:807-18.

18. The Diabetes Control and Complications Trial Research Group. The effect of intensive treatment of diabetes on the development and progression of long-term complications in insulindependent diabetes mellitus. N Engl J Med 1993;329:977-86.

19. Martens-Lobenhoffer J, Bode-Böger SM. Quantification of L-arginine, asymmetric dimethylarginine and symmetric dimethylarginine in human plasma: a step improvement in precision by stable isotope dilution mass spectrometry. J Chromatogr B 2012;904:140-3.

20. Versari D, Daghini E, Virdis A, Ghiadoni L, Taddei S. Endothelial dysfunction as a target for prevention of cardiovascular disease. Diabetes Care 2009;32:S314-21.

21. Henningsson R, Lundquist I. Arginine-induced insulin release is decreased and glucagon increased in parallel with islet NO production. Am J Physiol 1998;275:E500-6.

22. Jaźwińska-Kozuba A, Martens-Lobenhoffer J, Kruszelnicka 0, Rycaj J, Chyrchel B, et al. Opposite associations of plasma homoarginine and ornithine with arginine in healthy children and adolescents. Int J Mol Sci 2013;14:21819-32.

23. Järvisalo MJ, Raitakari M, Toikka JO, Putto-Laurila A, Rontu R, et al. Endothelial dysfunction and increased arterial intimamedia thickness in children with type 1 diabetes. Circulation 2004;109:1750-5. 
24. Ceriello A, Esposito K, Ihnat M, Thorpe J, Giugliano D. Long-term glycemic control influences the long-lasting effectof hyperglycemia on endothelial function in type 1 diabetes. J Clin Endocrinol Metab 2009;94:2751-6.

25. West MB, Ramana KV, Kaiserova K, Srivastava SK, Bhatnagar A. $\mathrm{L}$-arginine prevents metabolic effects of high glucose in diabetic mice. FEBS Lett 2008;582:2609-14.

26. Eid HM, Lyberg T, Arnesen H, Seljeflot I. Insulin and adiponectin inhibit the TNF $\alpha$-induced ADMA accumulation in human endothelial cells: the role of DDAH. Atherosclerosis 2007;194:e1-8.

27. Eid HM, Reims H, Arnesen H, Kjeldsen SE, Lyberg, T, et al. Decreased levels of asymmetric dimethylarginine during acute hyperinsulinemia. Metab Clin Exp 2007;56:464-9.
28. Marcoveccio ML, Widmer B, Dunger DB, Dalton RN. Effect of acute variations of insulin and glucose on plasma concentrations of asymmetric dimethylarginine in young people with type 1 diabetes. Clin Sci (Lond) 2008;115:361-9.

29. Haller MJ, Stein JM, Shuster JJ, Theriaque D, Samyn MM, et al. Pediatric Atorvastatin in Diabetes Trial (PADIT): a pilot study to determine the effect of atorvastatin on arterial stiffness and endothelial function in children with type 1 diabetes mellitus. J Pediatr Endocrinol Metab 2009;22: 65-8.

30. Beltowski J, Kedra A. Asymmetric dimethylarginine (ADMA) as a target for pharmacotherapy. Pharmacol Rep 2006;58: 159-78. 